\title{
CONTROVERSY
}

\section{Sedation of children for magnetic resonance imaging}

\author{
G R Lawson
}

Current British guidelines oppose the use of deep sedation by non-anaesthetists. ${ }^{12}$ but all reflect the needs and experience of adult practice. Despite this, and without reference to paediatric opinion, the Royal College of Surgeons of Edinburgh in 1993 stated: "Intravenous sedation is hazardous in children as the therapeutic margin between sedation and anaesthesia is very narrow. In view of this it should be administered only under very special circumstances." 2

At about the same time quite different conclusions were being reached within the paediatric guidelines produced by the American Academy of Pediatrics in $1985^{3}$ and revised in 1992. ${ }^{4}$ These guidelines stated: “ . . .deep sedation in children is an acceptable end point ..." and that it is "...not mandatory that deep sedation be supervised by an anaesthetist ...”.

For some time paediatricians have sought the optimal method to reduce the level of consciousness either to allow a procedure to be performed or to avoid the psychological sequelae of a painful or unpleasant procedure. With increasing emphasis on evidence based practice and risk management it was clearly important that this subject was approached with these factors in mind. Stephen Murphy wrote an article for this journal on paediatric sedation in 1997 , which focused on the issues of efficacy and safety. ${ }^{5}$

A highly respected paediatric anaesthetist wrote in September 1995 "Sedating children in order to carry out MRI scans is dangerous and inappropriate and general anaesthesia is the only (safe) choice" (Bray RJ, personal communication, 1995). This article will attempt to determine whether this view can be sustained and will assess the information regarding deep sedation in children undergoing magnetic resonance imaging (MRI) without repeating the arguments put forward in the previous article, ${ }^{5}$ which are taken as read.

\footnotetext{
Department of Paediatrics, Sunderland Royal Hospital, Kayll Road, Sunderland SR4 7TP, UK

G R Lawson

Correspondence to: Dr Lawson

email: g.r.lawson@ncl.ac.uk
}

a deeper level of sedation is required. may go to sleep with a feed or be adequately sedated with oral chloral hydrate. A recent
As movement interferes with effective MRI, a challenging problem is provided by patients unable to lie still. Conscious sedation is unable to guarantee patient compliance and therefore series of 1155 nurse led sedations for MRI at Great Ormond Street Hospital (London, UK) had a $1 \%$ failure rate for children weighing $5-10 \mathrm{~kg}$ with only $8 \%$ of this group needing intravenous in addition to oral sedative drugs. ${ }^{6}$ Children older than 7 years can often comply with instructions to remain still. Intravenous sedation is thus required for many of those between 1 and 7 years, and some older children with learning difficulties or claustrophobia; this was needed in almost $30 \%$ of children heavier than $10 \mathrm{~kg}$ in the aforementioned study. ${ }^{6}$

The nature of the MRI hardware precludes easy access to the patient whose head lies over one metre inside the housing of the electromagnetic coils of the scanner. This differentiates MRI scanning from other situations in which deep sedation can be used, such as minor procedures in the accident and emergency department or endoscopy in which immediate access to the child and its airway is not an issue. As a consequence, difficulty of access is the principal argument concerning the safety of deep sedation compared to general anaesthesia. Additionally, MRI requires specially designed equipment that can function within a powerful magnetic field, that does not degrade the image by interference, and that does not cause injuries from currents induced by strong magnetic fields. Although such specialised equipment is expensive, this should not be a significant consideration when safety is of paramount importance.

It is worth remembering that the definition of conscious sedation given by the American Academy of Pediatrics" is "a medically induced state of CNS depression in which communication is maintained so that the patient can respond to verbal command". Protective reflexes are preserved and the patient can independently maintain a patent airway. Meanwhile deep sedation is defined as "a medically induced state of CNS depression in which the patient is essentially unconscious, and so does not respond to verbal command". The patient breathes spontaneously but protective reflexes may be lost and the ability to maintain an airway is not assured. The potential complications of deep sedation include hypoventilation, apnoea, airway obstruction, aspiration, hypotension, bradycardia, and increased intracranial pressure. A spectrum of sedation may exist, but with oral 
and intramuscular regimens in older children it is possible to move from conscious sedation to deep sedation without ready recognition. Intravenous sedation is more predictable in this group as it has an immediate effect and is much less reliant on other factors such as absorption. However, it has also been suggested that there are varying levels of deep sedation at the end of which is an overlap with general anaesthesia. ${ }^{4}$

\section{Safe practice guidelines}

Present American paediatric guidelines ${ }^{4}$ augmented by a literature search suggests that if deep sedation is required then it should be performed by someone:

- who is working to an accepted guideline

- with sole responsibility for the sedation

- who has been trained to an acceptable level (such as, Advanced Paediatric Life Support provider status)

- who is familiar with the drugs, dosages, monitoring equipment, and requirements of the procedure

- who is supported by other skilled staff such as a children's nurse.

Contraindications to sedation exist ${ }^{67}$ and include:

- potential airway obstruction-for example, sleep apnoea

- respiratory centre abnormalities-for example, brain stem tumours

- respiratory centre desensitised to carbon dioxide-for example, conditions with chronically raised $\mathrm{PaCO}_{2}$

- renal or hepatic dysfunction leading to altered drug kinetics

- conditions in which a rise in $\mathrm{PaCO}_{2}$ would be detrimental-for example, raised intracranial pressure

- conditions with high risk of pulmonary aspiration of gastric contents.

Children should be prepared in a similar way

to a child undergoing anaesthesia:

- informed consent for sedation taken

- children fasted by withholding milk and solids for four hours before sedation

- reliable intravenous access essential before, during, and after the procedure if intravenous drugs are used.

There has been much debate over appropriate drugs and their dosage, and those who sedate children will have their favourite regimens. From the literature it appears important that the person administering the drugs is familiar with them, and that cocktails of more than two drugs are to be avoided because of the unpredictability of drug interactions and the increased incidence of important side effects. ${ }^{8} 9$ Minimum doses should be used to achieve the necessary level of sedation and yet it is preferable to give a reasonable bolus of drug rather than attempting to titrate the dose by small repeated increments over a prolonged period.

Chloral hydrate is an extremely useful and safe oral drug and can be used with good effect in children up to $10 \mathrm{~kg} .{ }^{6}$ For those requiring intravenous drugs midazolam is a short acting benzodiazepine that is frequently used for sedation in adults and children alike. In general it "seems to have much greater potential for
Table 1 Usual and maximum dosages for commonly used sedatives and their antidotes

\begin{tabular}{lll}
\hline Drug & Dosage & Maximum dosage \\
\hline Chloral hydrate & $50 \mathrm{mg} / \mathrm{kg}(<5 \mathrm{~kg})$ & \\
& $\begin{array}{l}100 \mathrm{mg} / \mathrm{kg} \\
(5-10 \mathrm{~kg})\end{array}$ & \\
& $0.1 \mathrm{mg} / \mathrm{kg}$ & $0.75 \mathrm{mg} / \mathrm{kg}$ or \\
Midazolam & $15 \mathrm{mg}$ total \\
& $1 \mathrm{mg} / \mathrm{kg}$ & $2 \mathrm{mg} / \mathrm{kg}$ \\
Pethidine & $0.5 \mathrm{mg} / \mathrm{kg}$ & $20 \mu \mathrm{g} / \mathrm{kg}$ \\
Pentazocine & $10 \mu \mathrm{g} / \mathrm{kg}$ & \\
Flumazenil & $5 \mu \mathrm{g} / \mathrm{kg}$ & \\
Naloxone & & \\
\hline
\end{tabular}

respiratory depression in the elderly than in children"10; however, it should be used with extreme caution with fentanyl because of adverse interactions. It also has increased sedative action when given at the same time as erythromycin. It is often combined with pethidine or pentazocine, which potentiate its sedative effect. Flumazenil is the antidote to benzodiazepines and naloxone for opiates but they should not be routinely used to "reverse" the sedation. Their effect is generally shorter than the potential effect of the sedative drugs, which could lead to children becoming more sedated following discharge. Table 1 shows the usual and maximum dosages for commonly used sedatives and their antidotes.

After giving the sedative drugs it is helpful if the head is extended to avoid forward flexion, which may result in airway obstruction. Thereafter, monitoring the patient during and following the procedure is the cornerstone of safe practice. ${ }^{4}$ Pulse oximetry and close physical observation are essential. During sedation, oxygen saturation should stay above $93 \%$. Observations including time of administration of drugs, time of achievement of sedation, and time to recovery should be recorded. Vital signs such as level of consciousness, pulse, respiratory rate, and oxygen saturation readings should be taken at five minute intervals during the procedure and any adverse events must be recorded.

Resuscitation equipment should be readily available and should include:

- oxygen

- bag and mask with oral airways

- suction apparatus

- intubation tubes and laryngoscope.

After the scan, observations should be continued and recorded until recovery of consciousness has been achieved and the child is responding to verbal commands in an appropriate way. Resuscitation equipment should be readily available in the recovery area.

\section{Adverse events with sedation in children}

Several case reports of adverse events are to be found in the literature; however, none provides data on the denominator, which would give an indication of the relative risk of sedation. In a poster publication by Coté et al, American data over 28 years for patients younger than 21 years was analysed..$^{11}$ From 69 reports (39 patients $<4$ years) there were 52 deaths ( 22 in patients $<4$ years). Fourteen occurred in association with "computed tomography/MRI/radiology". Of the common causes at all ages, drug overdose ( $n=38$ ) was most frequent followed 
by inadequate monitoring $(n=25)$, followed by premature discharge $(\mathrm{n}=14)$, inadequate help $(n=9)$, drug interaction $(n=6)$, and drug error $(n=4)$. Twenty six occurred in hospital. No denominator is stated and an acknowledgement is given regarding the inherent limitations of such data.

\section{General anaesthesia}

Advantages may be achieved by using general anaesthesia instead of deep sedation. There should be fewer failures and there may be a faster turn round. However data are not available to determine whether it is a safer than deep sedation. The disadvantages of general anaesthesia include the need for dedicated anaesthetic equipment and a greater availability of paediatric anaesthetists. ${ }^{6}$ Indeed, it could be argued that children could have significant delays in gaining results from important investigations through long waiting lists for procedures carried out under general anaesthesia rather than sedation. This in turn might lead to an adverse outcome, which might have been avoided by a more timely investigation done under sedation.

\section{Conclusion}

The final argument as to whether deep sedation in children is safe for MRI is finely balanced. The only data on significant adverse reactions other than anecdotal evidence are offered in a poster by the same author ${ }^{11}$ who, in reviewing the subject in 1994, concluded that safe sedation was possible in children providing previously stated guidelines were followed. ${ }^{10}$ His poster's conclusion is not that sedation be abandoned but that "Our specialty's [anaesthesiology] involvement in the development and enforcement of sedation guidelines within institutions is critical." Indeed, if the guidelines for safe practice had been followed, most if not all of the adverse events might have been avoided.

Ferguson and Ball, ${ }^{12}$ themselves senior registrars in anaesthesia, stated " . . but in special circumstances or complex patients ... the paediatrician should feel comfortable in approaching a paediatric anaesthetist for advice and assistance." British paediatric anaesthetists are opposed to deep sedation in principle but do not have objective evidence to unequivocally support their case. I suspect they have grave misgivings about any situation in which they do not have good control of the airway. However, their dogmatic stance is not contributing to the debate and is inhibiting otherwise good relationships based on a common desire to achieve what is best for the child.

If one accepts that deep sedation can only be acceptable if it causes no more deaths than general anaesthesia and that one death or episode of serious morbidity from sedation is unacceptable if anaesthesia is safer, then resolution of this argument must come from a national confidential inquiry into adverse events. As part of this, the denominator for all children undergoing sedation or anaesthesia must be known if these figures are to be of value. Until then a pragmatic stance would seem to be to allow deep sedation for MRI and other procedures, providing that the previously stated guidelines are observed closely. Enter the National Institute for Clinical Excellence police and some firm clinical governance.

I acknowledge the help offered by Drs Stephen Chapman and Stephen Murphy of Birmingham Children's Hospital who gave constructive criticisms of this paper based on their considerable experience of sedating children. A guideline for considerable experience of sedating children. A guideline for sedating children for MRI based on this article and its 作 G.R.LAWSON@NCL.AC.UK> and may be modified to individual departmental needs.

1 Royal Colleges of Anaesthetists and Radiologists. Report of a joint working party. Sedation and anaesthesia in radiology. London: Royal Colleges of Anaesthetists and Radiologists, 1992.

2 Royal College of Surgeons of England. Commission on the provision of surgical services. Report of the working party on guidelines for sedation by non-anaesthetists. London: Royal College of Surgeons, 1993

3 American Academy of Pediatrics. Committee on Drugs, Section on Anesthesiology. Guidelines for the elective use of conscious sedation, deep sedation, and general anaesthesia in pediatric patients. Pediatrics 1985;76:317-21.

4 American Academy of Pediatrics. Committee on Drugs. Guidelines for monitoring and management of pediatric patients during and after sedation for diagnostic and therapeutic procedures. Pediatrics 1992;89:1110-15

5 Murphy MS. Sedation for invasive procedures in paediatrics. Arch Dis Child 1997;77:281-6.

6 Sury MRJ, Hatch DJ, Deeley T, Dicks-Mireaux C, Chong WK. Development of a nurse-led sedation service for paediatric magnetic resonance imaging. Lancet 1999;353: diatric mag $1667-71$.

7 Shepherd JK, Hall-Craggs MA, Finn JP, Bingham RM. Sedation in children scanned with high-field magnetic resonance; the experience at the Hospital for Sick Children, Great Ormond Street. Br F Radiol 1990;63:794-7.

8 American Academy of Pediatrics. Reappraisal of lytic cocktail/demerol, phenergan, and thorazine (DPT) for the sedation of children. Pediatrics 1995;95:598-602

9 Nahata MC, Clotz MA, Krogg EA. Adverse effects of meperidine, promethazine, and chlorpromazine for sedation in pediatric patients. Clin Pediatr 1985;24:558-60.

10 Coté CJ. Sedation for the pediatric patient. Pediatr Clin $N$ Am 1994;41:31-58.

11 Coté CJ, Alderfer RJ, Notterman DA, Fanta KB. Sedation disasters: adverse drug reports in pediatrics-FDA, USP and others [abstract]. Anesthesiology 1995;83:A1183.

12 Ferguson S, Ball AJ. Sedation and sedative drugs in paediatrics. Br f Hosp Med 1996;55:611-15.

\section{Commentary}

General anaesthesia undoubtedly allows MRI to be carried out in anxious children, but sedation is sometimes seen as an acceptable alternative, particularly in the United States. Conscious sedation is impractical in a noisy environment and deep sedation is necessary, ${ }^{12}$ in spite of official disapproval. ${ }^{3}$ Deep sedation usually involves a bolus of an oral hypnotic, which may need to be topped up with an intravenous tranquilliser or opioid. During the scan the child is largely hidden and out of reach, often with depressed ventilation and impaired airway reflexes, and without any airway maintenance device in place, a situation with which most anaesthetists would feel uncomfortable. Anaesthesia starts with a rapid intravenous or gaseous induction, followed by some method of securing the airway. Anaesthesia is maintained for as long as necessary by using some combination of gases, volatile agents or intravenous drugs. Unlike sedation, deepening the level of consciousness or dealing with respiratory depression or apnoea is simple, almost immediate and not disruptive to the scan. To decide between sedation and anaesthesia it will be helpful to compare how they meet the requirements for scanning.

RELIABILITY

General anaesthesia produces an immobile patient who will stay unconscious until the end 
of the procedure. Sedation is less predictable and it is accepted that there is a failure rate of between $5 \%$ and $15 \% .^{24-6}$

\section{RAPIDITY OF TURNOVER}

The induction of anaesthesia is relatively quick but sedation has a longer and more variable onset and offset ${ }^{5}$ during which the child must be observed. The scanner may be used for other patients in the meantime but overall the turnover is probably slower.

CosT

General anaesthesia requires an anaesthetist, an assistant, anaesthetic and monitoring equipment, and drugs. Sedation requires a sedationist, an assistant, monitoring and resuscitation equipment, and drugs. The Association of Anaesthetists recommends that monitoring standards should be the same for sedation and anaesthesia: continuous ECG and pulse oximetry, some means of measuring respiration such as end tidal $\mathrm{CO}_{2}$ monitoring, frequent blood pressure estimations, and inspired oxygen concentration monitoring if the patient is breathing from a gas delivery system. ${ }^{7}$ The cost of anaesthetic equipment cannot be avoided if the children who failed to be sedated are dealt with on the same site. Comparable grades of paediatrician, radiologist, and anaesthetist cost the same although nurse sedationists cost less. Taking into account sedation failures and a slower turnover it is likely that there is little overall saving unless the radiologist doubles up as the sedationist, a practice not generally approved. ${ }^{178}$

\section{AVAILABILITY}

Difficulties obtaining anaesthetists may have provided the motivation for seeking an alternative, but in most centres the demand for MRI anaesthetic time is a small fraction of the surgical demand and should be easy to deal with as long as the scans are batched. Paediatricians or radiologists are unlikely to be any more available than anaesthetists.

SAFETY

Sedation is often viewed as less dangerous than general anaesthesia. It is hard to imagine why long acting drugs administered in a less controlled fashion by less skilled and experienced personnel without the provision of airway maintenance equipment and frequently with inadequate monitoring should be thought safer. The mortality from general anaesthesia alone is about 1 in 160000 administrations, ${ }^{9}$ most perioperative deaths being caused by the patient's surgical condition. The mortality from sedation is less certain as most prospective series are too small to produce reliable data. The largest UK series comprised 14149 upper gastrointestinal endoscopies, mainly in adults. ${ }^{10}$ There were five deaths (not including those from perforation or bleeding), a rate of 1 in 2800; almost 60 times the mortality of general anaesthesia. A comparison between sedation and anaesthesia for upper gastrointestinal endoscopy in children showed a higher incidence of desaturations and arrhythmias in the sedation group. ${ }^{11}$ As well as Coté et al's collection of sedation disasters, ${ }^{12}$ there are many other reports of serious incidents, ${ }^{111^{13-15}}$ one mentioning nine deaths and 18 episodes of respiratory arrest occurring during sedations carried out by 129 radiologists. ${ }^{15}$

There seems no reason why the standard of care expected by the courts during sedation should be any lower than that during anaesthesia, and the responsibility for sedations delegated to nurses or trainee radiologists or paediatricians, who may lack the authority to interrupt a scan, ${ }^{16}$ will remain with the delegating consultant. ${ }^{17}$ Recently, in the UK a child received almost $£ 4$ million in compensation for hypoxic brain damage ${ }^{18}$ and there is the ever present fear of a manslaughter charge when a patient dies. ${ }^{19}$ Safety would seem to be a decisive argument in favour of general anaesthesia.

\section{CONCLUSIONS}

It may be possible to use deep sedation to produce satisfactory conditions for children having MRI scans, but general anaesthesia is safer and more reliable. Just because something is possible does not mean that it is best practice, and that is what we should be providing for our children.

Consultant Anaesthetist, Royal Victoria Infirmary, R J BRAY

Nerwcastle upon Tyne, UK

1 Maxwell LG, Yaster M. The myth of conscious sedation. Arch Pediatr Adolesc Med 1996;150:665-7.

2 Sury MRJ, Hatch DJ, Deeley T, Dicks-Mireaux C, Chong WK. Development of a nurse-led sedation service for paediatric magnetic resonance imaging. Lancet 1999;353: 1667-71.

3 Sedation and anaesthesia in radiology. Report of a joint working party. London: The Royal College of Anaesthetists and The Royal College of Radiologists, 1992.

4 Malviya S, Voepel-Lewis T, Tait AR. Adverse events and risk factors associated with the sedation of children by nonanesthesiologists. Anesth Analg 1997;85:1207-13.

5 Squires RH, Morriss F, Schluterman S, Drews B, Galyen L, Brown KO. Efficacy, safety, and cost of intravenous sedation versus general anaesthesia in children undergoing endoversus general anaesthesia in children undergoing
scopic procedures. Gastrointest Endosc 1995;41:99-104.

6 Hollman GA, Elderbrook MK, Van Den Lagenberg B. Results of a pediatric sedation program on head MRI scan success rates and procedure duration times. Clin Pediatr success rates and

7 Recommendations for standards of monitoring during anaesthesia and recovery. London: The Association of Anaesthetists of Great Britain and Ireland, 1994.

8 Practice guidelines for sedation and analgesia by nonanesthesiologists. A report by the American Society of Anesthesiologists task force on sedation and analgesia by non-anesthesiologists. Anesthesiology 1996;84:459-71

9 Buck N, Devlin HB, Lunn JN. The report of a confidential enquiry into perioperative deaths. London: Nuffield Provincial Hospitals Trust, 1987.

10 Quine MA, Bell GD, McCloy RF, Charlton JE, Devlin HB, Hopkins A. Prospective audit of upper gastrointestinal endoscopy in two regions of England: safety, staffing, and sedation methods. Gut 1995;36:462-7.

11 Lamireau T, Dubreuil M, Daconceicao M. Oxygen saturation during esophagogastroduodenoscopy in children: general anesthesia versus intravenous sedation. $\mathcal{F}$ Pediatr Gastroenterol Nutr 1998;27:172-5.

12 Coté CJ, Alderfer RJ, Notterman DA, Fanta KB. Sedation disasters: adverse drug reports in pediatrics [abstract] Anesthesiology 1995;83:A1183.

13 Nahata MC, Clotz MA, Krogg EA. Adverse effects of meperidine, promethazine, and chlorpromazine for sedation in pediatric patients. Clin Pediatr 1985;24:558-60.

14 D'Agostino J, Terndrup TE. Comparative review of the adverse effects of sedatives used in children undergoing outpatient procedures. Drug Saf 1996;14:146-57.

15 Cohen MD. Pediatric sedation. Radiology 1990;175:611-12. 16 Strain JD, Campbell JB, Harvey LA, Foley LC. IV nembutal: safe sedation for children undergoing CT. Am $\mathcal{F}$ Roentgenol 1988;151:975-9.

17 Anaesthesia in Great Britain and Ireland. A physician only service. London: The Association of Anaesthetists of Great service. London: The Association

18 Brain damage girl awarded record $£ 3.9$ million. The Times, 17 Nover

19 Doctor jailed over fatal mix-up. The Times, 30 July 1999. 